\title{
ONIARE
}

\section{El impacto de la cultura del diseño en el éxito de las organizaciones}

Fecha de recepción: 18 de septiembre de 2017

Fecha de aprobación: 20 de noviembre de 2017

\author{
Alexandra Forero ${ }^{l}$ \\ Universidad EAN \\ aforerod.d@universidadean.edu.co \\ Luz Amparo Acosta Salas ${ }^{2}$ \\ Universidad EAN \\ lacosta@universidadean.edu.co
}

DOI: https://doi.org/10.21158/23823399.v5.n0.2017.2006

Cómo citar este artículo/ To reference this article/Comment citer cet article/ Para citar este artigo: Forero, A. y Acosta Salas, L. A. (2017). Factores clave de éxito en proyectos de software en el sector financiero colombiano. Revista Ontare. 5, p 49 - 72.

\section{Resumen}

El Design Thinking, o pensamiento de diseño, más que una metodología es una filosofía de trabajo usada en procesos de innovación, que utiliza herramientas y metodologías que, por lo general, usan diseñadores para resolver problemas complejos (Mora, 2012). De igual forma permite centrarse en el descubrimiento de insights, un proceso centrado en el usuario que hace énfasis en la observación, la colaboración, el aprendizaje rápido y la visualización de ideas que se complementa con la filosofía de desarrollo de clientes por medio del prototipado rápido y la construcción de las características mínimas del producto o servicio (Mora, 2012). El desarrollo de un modelo para la medición de este impacto dentro de las culturas organizacionales fue el eje central de esta investigación.

\section{Palabras clave}

Innovación; pensamiento de diseño; cultura; cultura del diseño; organización.

1 Universidad EAN. Correo electrónico: aforerod.d@universidadean.edu.co.

2 Ingeniera de sistemas, con especialización en Gerencia de Tecnología de la Universidad EAN. ORCID: https://orcid.org/0000-0003-0409-203X 


\title{
The impact of the design culture on organizational success
}

\begin{abstract}
The Design Thinking, rather than a methodology is a work philosophy used in innovation processes, which uses tools and methodologies that generally, designers use to solve complex problems (Mora, 2012). It also allows focusing on the discovery of insights, a user-centered process that emphasizes observation, collaboration, rapid learning, and visualization of ideas, that is complemented by the philosophy of customer development through rapid prototyping and the construction of the minimum characteristics of the product or service (Mora, 2012). The development of a model for the measurement of this impact within organizational cultures was the central axis of this research.
\end{abstract}

\section{Keywords}

Innovation; design thinking; culture; design culture; organization.

\section{L'impact de la culture du design dans le succès des organisations}

\section{Résumé}

La pensée conceptuelle, ou design thinking, est davantage une philosophie qu'une méthodologie de travail qui est utilisée lors des processus d'innovation grâce à des outils et méthodologies dont les concepteurs se servent pour résoudre des problèmes complexes (Mora, 2012). La pensé conceptuelle permet notamment de se focaliser sur la découverte d'idées, processus centré sur l'utilisateur mettant l'accent sur l'observation, la collaboration, l'apprentissage rapide et la visualisation des idées, complété en cela par la philosophie du développement client, le prototypage et la construction des caractéristiques minimales du produit ou du service (Mora, 2012). Le développement d'un modèle de mesure de cet impact au sein des cultures organisationnelles a été l'axe central de cette recherche.

\section{Mots clefs}

Innovation; design thinking; culture; culture du design; organisation. 


\section{O impacto da cultura do desenho no sucesso das organizações}

\section{Resumo}

O Design Thinking, ou pensamento de desenho, mais que uma metodologia é uma filosofia de trabalho usada em processos de inovação que utiliza ferramentas e metodologias que, em geral, usam desenhistas para resolver problemas complexos (Mora, 2012). De igual forma permite concentração na descoberta de insights, um processo centrado no usuário que faz ênfase na observação, na colaboração, na aprendizagem rápida e na visualização de ideias que se complementam com a filosofia de desenvolvimento de clientes por meio do prototipado rápido e da construção das características mínimas do produto ou serviço (Mora, 2012). $O$ desenvolvimento de um modelo de medição deste impacto dentro das culturas organizacionais foi o eixo central desta pesquisa.

\section{Palavras-chave}

Inovação; pensamento de desenho; cultura; cultura do desenho; organização. 


\title{
1. Introducción
}

\begin{abstract}
Igunos autores afirman que el pensamiento de diseño o design Athinking (DT) consiste en un conjunto de herramientas y metodologías utilizadas para la solución de problemas complejos ${ }^{3}$. Según Dahi (2015), es una actitud humana-centrista abierta que se desarrolla para crear e implementar una solución alternativa holística de problemas complejos: «Actualmente, la mayoría de los desafíos son complejos, los múltiples números de factores y sus relaciones, que se manifiestan en sistemas independientes, cambian el carácter de estos retos todo el tiempo ${ }^{4} \gg$. Kaltenbach (2013) asegura que, sorprendentemente, los sistemas de pensamiento (systems thinking) van de la mano con el DT, así sea que se planee o no. Por tanto, es esencial definir o redefinir los problemas y así revelar cuál es la verdadera esencia de este.
\end{abstract}

Otros autores explican que el pensamiento de diseño es simplemente una «actitud» con la que se afrontan problemas de alta incertidumbre y, por esto, es ideal para el desarrollo de la innovación. «El DT es una aproximación diferente para resolver problemas e incentivar la innovación. No solo puede ser aplicada por diseñadores, sino que puede ser también aplicada en cualquier otra rama del conocimiento. Los pilares del DT son las personas, la creatividad y la experimentación ${ }^{5} . »$ (Dahi, 2015).

3 Según Rittel (1960), los problemas complejos o wicked problems son «una clase de problemas sociales que están mal formulados, donde la información es confusa, hay muchos clientes y responsables de toma de decisiones con valores conflictivos, y las ramificaciones de todo el sistema son completamente conflictivas».

4 La traducción es nuestra.

5 La traducción es nuestra. 
Algunos, incluso, afirman que este conjunto de conocimientos, métodos $\mathrm{y}$ actitudes puede ser la diferencia entre una empresa tradicional y una empresa que innova de manera espontánea. El DT, principalmente, es un proceso de innovación, es la parte «borrosa» y un gran método con el que se pueden descubrir necesidades ${ }^{6}$ insatisfechas y crear la oferta de nuevos productos y servicios, sin mencionar la transformación de negocios por medio de la solución de problemas complejos (Lockwood, 2009).

\section{Desarrollo}

Tras revisar más de cincuenta publicaciones, se definió un modelo como base que representa los principales constructos asociados con el desarrollo de una cultura de diseño y la medición de su impacto.

La figura 1 muestra cómo con la cultura de diseño se puede lograr un éxito organizacional, teniendo en cuenta los conceptos de diseño e innovación, que tienen una relación directa con la cultura de diseño y, por ende, con el éxito organizacional. La cultura organizacional también debe estar relacionada con los conceptos de diseño, innovación y cultura de diseño para alcanzar el éxito de la organización.

6 La necesidad es el factor motivacional más importante en la configuración del entorno al que el diseño, por supuesto, no es ajeno. La necesidad es resultado de la conciencia de alguna deficiencia que cuando es satisfecha produce sensaciones gratificantes: goce, placer, bienestar, relajamiento, etc. El diseño satisface necesidades que concreta por medio del uso de objetos y la configuración de significados (Colomer, 2012). 
Figura 1. La cultura del diseño y el éxito organizacional

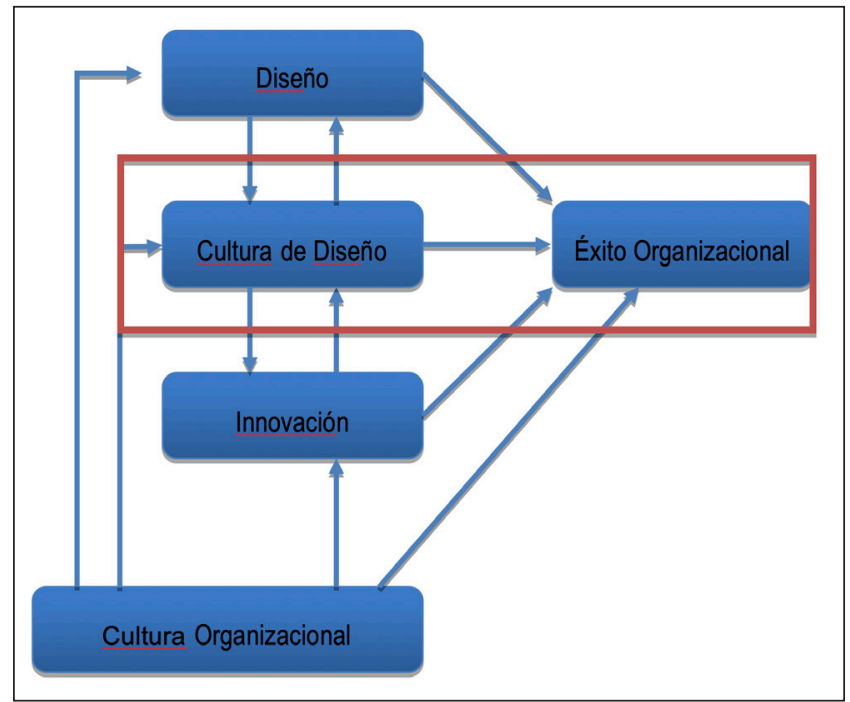

Fuente. Elaboración propia.

Bremner y Rodgers (2013) hablan del diseño como un «espacio dinámico y desafiante en el que continuamente se están desdibujando los límites disciplinarios profesionales ${ }^{7} »$. Por esto, el diseño se ha convertido en un concepto que abarca diferentes ámbitos tangibles e intangibles (Lautor, 2008). Rams (1991) asegura que el diseño debe estar involucrado con «la economía y con la ecología, el tráfico y las comunicaciones, los productos y servicios, la tecnología e innovación, la cultura y civilización, con aspectos sociales psicológicos, médicos, físicos, ambientales y políticos; y con todas las formas de organización social ${ }^{8}$ » (p. 77). El diseño ahora abarca múltiples perspectivas disciplinarias —es decir, se caracteriza por ser multidisciplinaria - y conlleva actividades transversales. Con lo cual ayuda a unificar resultados sostenibles y sustanciales, incluso en desarrollar nuevos conceptos y metodologías

7 La traducción es nuestra

8 La traducción es nuestra 
basadas en el diseño, como el pensamiento de diseño o DT, que se convierte en una ficha fundamental para la innovación.

Existen indicadores que permiten medir el diseño como herramienta (i. e. rendimiento, productividad, una fuerte y positiva cultura corporativa, trabajo en equipo, buenas comunicaciones, toma de decisiones, recompensas, entre otras, que aportan resultados positivos de gran importancia en las organizaciones (Reyes, Rodríguez y Pazcagaza, 2014).

Estudios como Dsg-II Strategic blueprint of the designsingapore initiative (Design Singapore Council, Ministry of Information, Communications and the Arts, 2000) o El impacto económico del diseño en las empresas de Cataluña (Observatorio Diseño y Empresa [BCDO], 2009) demuestran resultados favorables tanto en el incremento en el producto bruto interno (PBI) como en la rentabilidad de las empresas y la cultura corporativa, debido a las inversiones que hacen estas incorporando el diseño y potenciando el valor de este como medio para innovar en todos los aspectos — productos, servicios y clima organizacional-.

Se considera entonces el diseño como parte integral del «triple resultado», que consiste en generar beneficios económicos, sociales y ambientales. Borja (2006) ha investigado el desempeño del diseño por años, y plantea que este tiene cuatro poderes: como diferenciador, integrador, transformador o, simplemente, un buen negocio.

De esta manera, el uso del diseño como un proceso, una forma de interacción, un resultado o un recurso permite acercarse al objetivo de construir sistemas y procesos por medio de metodologías de diseño y de promover el estatus del diseño como una fuerte competencia en la organización. El valor real del diseño está en descubrir y resolver todo tipo de problemas. El diseño ha logrado un reconocimiento como parte generadora de la innovación. Se pueden encontrar tres grandes interpretaciones del diseño en el contexto de la innovación: 
El diseño como elemento tangible: una silla, un vehículo, una lámpara.

El diseño como actividad creativa: una actividad generadora de ideas.

El diseño como proceso con el que la información se transforma en un elemento tangible: el diseño como generador de ideas nuevas, su conceptualización y materialización (Carrió, 2006, p. 150) (Figura 2).

Figura 2. Diferenciación entre los diversos niveles de comprensión del diseño en el proceso de innovación

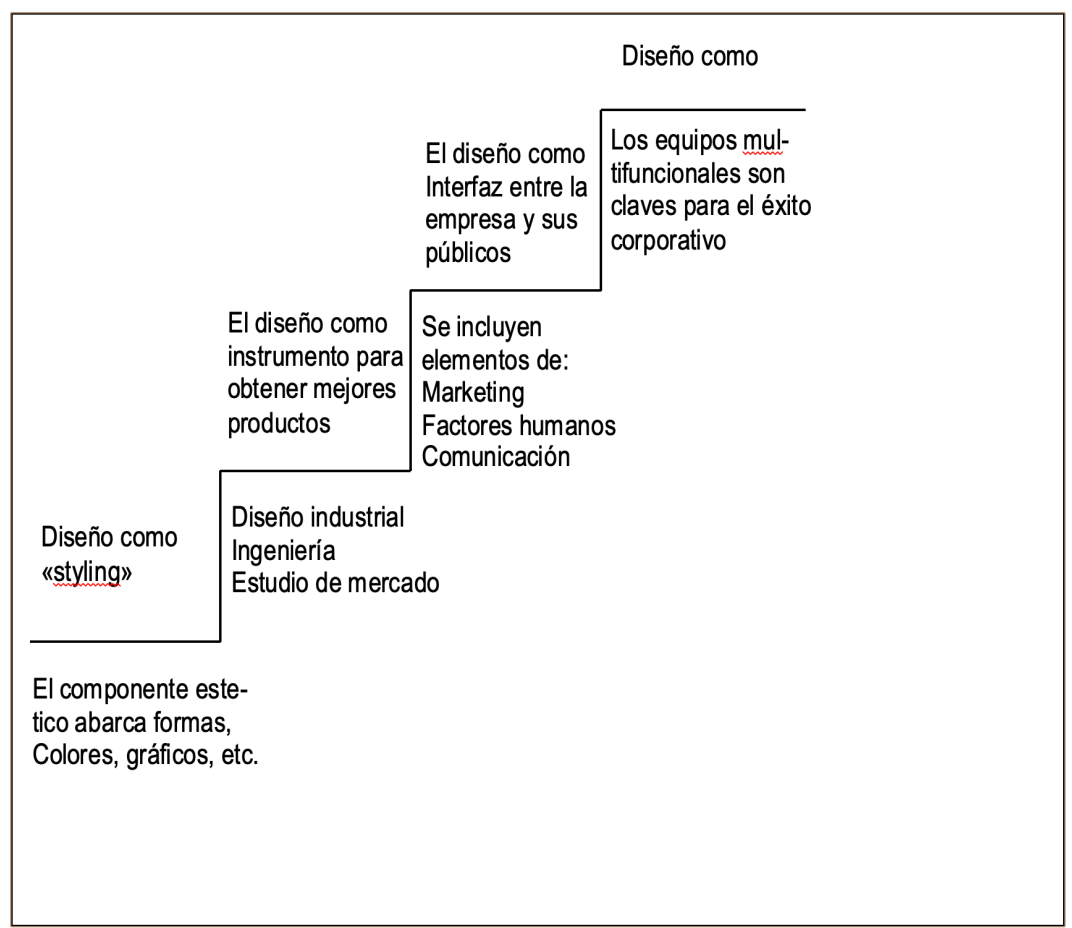

Fuente. Fairhead, 1988.

De este modo, la innovación, a través del diseño y sus procesos, genera un cambio tanto en la economía de las empresas como en la cultura organizacional de estas y un éxito corporativo. «Se puede afirmar, pues, que existe un amplio consenso de que la innovación es imprescindible 
para obtener nuevas ganancias de productividad que faciliten, entre otros objetivos, la mejora de la competitividad en las empresas» (Colomer, 2012, p. 61).

Sin embargo, «lo que no se mide, no existe», así que existen diferentes modelos y métodos de análisis para la medición de la innovación. Debido a que este estudio se centró en medir el impacto de una cultura de diseño en las organizaciones, se hizo hincapié en tener como referentes modelos o métodos que midan la cultura de innovación dentro de las empresas. Las encuestas sobre innovación pueden proporcionar datos acerca de estos temas, ya que la teoría de la innovación trata, precisamente, de la medición y del diseño de la teoría del conocimiento. (Colomer, 2012).

Deshpande y Webster (1989) realizaron una extensa revisión de más de cien artículos sobre comportamiento organizacional, sociología y antropología, tras lo cual definieron la cultura organizacional cómo «el patrón de valores y creencias compartidas que ayudan a los individuos a entender el funcionamiento de una organización y de este modo facilitarles la adaptación a las normas de comportamiento de la organización ${ }^{9}$ (p. 4). Las ideas predominantes de una empresa, los valores, las actitudes y las creencias guían el camino de cómo sus empleados piensan, sienten y actúan.

Por otro lado, la literatura sobre la innovación de la organización se centra en el papel de las estructuras organizativas, de los procesos de aprendizaje y de la adaptación a los cambios en la tecnología y el entorno institucional y de los mercados. La organización de una empresa puede incidir en la eficacia de sus actividades innovadoras, dado que algunas de estas estructuras organizativas se adaptan mejor a determinados entornos (Lam, 2004). Al introducir nuevos procesos o metodologías dentro de la cultura de la organización, los cambios se van a ver representados en las

9 La traducción es mía. 
actitudes tanto de los empleados como de los líderes y, por ende, en su rendimiento y competitividad.

Las innovaciones en la organización del lugar de trabajo implican la introducción de nuevos métodos de atribución de responsabilidades y del poder de decisión entre los empleados para la división del trabajo en el seno de los servicios y entre los servicios - y las unidades organizativas - de la empresa, así como nuevos conceptos de estructuración, en particular, la integración de distintas actividades (Colomer, 2012). Un proceso del diseño como el pensamiento de diseño o DT delega a los empleados de las empresas mayor autonomía de toma de decisiones y los anima a comunicar sus ideas. Para ello, se puede descentralizar el control de gestión y las actividades del grupo o crear equipos formales o informales en los que cada empleado goce de una mayor flexibilidad (Colomer, 2012).

Al alinear la cultura corporativa con el diseño, se crea una estrategia imperativa, porque la cultura es el elemento esencial para la manera en que las colaboraciones ocurren entre los equipos de trabajo, en las organizaciones y en medio de las corporaciones y el mercado. De acuerdo con el experto en liderazgo de organizaciones Edgar Papke, es importante interpretar la cultura y considerar que los motivadores de los comportamientos humanos —necesidad de inclusión, competencias y apertura - integran de la mejor manera al diseño dentro de las empresas (Lockwood, 2009).

El diseño es una herramienta de innovación que debe enfocarse en el presente y proyectar ideas en el futuro teniendo siempre en cuenta la tradición de la organización, sus actitudes, experiencias y valores, tanto personales como culturales. En especial, en las pequeñas y medianas empresas, un valor sobre el que fundamentar el diseño es el conocimiento singular de cada empresa, o sea, en su cultura organizacional (Colomer, 2012). 
El diseño puede actuar como transmisor de los diferentes factores organizacionales de la empresa (Hofstede, Neuijen, Ohayv y Sanders, 1990), entre los que se identifican:

\subsection{Los valores:}

a. Sistema de valores: los valores deseados que sustentan las estrategias, que no deben ser impuestas, sino que se guiarán para ser compartidas por todo el personal de la organización, por lo cual se ha de trabajar en este sentido (Colomer, 2012).

\subsection{Los rituales:}

a. Hábitos de trabajo: tipo de nivel de actividades fundamentales dentro del trabajo mismo, cómo se hacen las cosas en la organización, cómo se percibe y trata al cliente, cómo se elaboran los informes y los productos, cómo se ofrece el servicio, entre otros. Cómo se comparten o no los resultados del trabajo desde su inicio a fin, cómo se comportan los canales formales e informales de transmisión de hábitos (Colomer, 2012).

b. Ritos y ceremonias: aspectos que rodean el trabajo, cómo se incentiva, cómo se llama la atención, cómo se influye en el comportamiento. Por ejemplo: asambleas, reuniones, procesos de selección y de evaluación, proceso de aprendizaje, orientación en que se toman las decisiones (Colomer, 2012).

\subsection{Los héroes:}

a. Características de los directivos: orientación en la toma de decisiones, vías que utilizar en la búsqueda de soluciones, conocimientos, autonomía, cómo expresar el poder, coeficiente emocional, etc. (Colomer, 2012). 


\subsection{Los símbolos:}

a. Organización, comunicación e información interna: cómo es la organización, los sistemas de información, la comunicación formal vertical y horizontal, su estructura organizativa y su congruencia con la estrategia, cómo apreciar los cambios en el clima cuando hay disfunción, etc.

Estos factores organizacionales son los que representan la cultura corporativa de la empresa, se pueden influenciar por las metodologías del diseño y generar una cultura.

Adecuar los factores organizacionales de la empresa a la estrategia de la empresa debe permitir esto, que se propaguen por todas sus áreas funcionales en mayor o menor medida. Esto generará un cambio del diseño de los signos de identidad y por tanto la cultura corporativa, de lo contrario, la imagen resultante no responderá a la realidad que se quiere transmitir al mercado (Colomer, 2012). Se debe producir una relación coherente entre los factores organizacionales de la empresa, la estrategia y los procesos de diseño para crear una cultura fuerte y competitiva.

Así, se puede concluir que el diseño y la innovación son elementos que deben ir siempre integrados y siempre van de la mano. Aunque la cultura organizacional también se verá afectada si alguno de estos cambia o deja de estar.

\section{Cultura de diseño}

Teniendo como preámbulo estas recopilaciones, revisiones y análisis bibliográficos, se decide usar el término cultura de diseño como aquel elemento que se puede aplicar dentro de las organizaciones para crear una cultura basada en herramientas y procesos de diseño y generar un cambio en el modo de pensar y actuar de los empleados y líderes. 
En la búsqueda del término cultura de diseño, las referencias se limitan a artículos y libros como el de Julier (2010), quien utiliza el término para hacer énfasis en los procesos creativos que llevan a cabo los diseñadores cuando van a desarrollar un producto. Sin embargo, en esta investigación, la cultura de diseño hace énfasis en el uso de metodologías como el pensamiento de diseño o DT concebido como una herramienta que está siendo utilizada para crear un cambio y una manera de resolver problemas complejos en las organizaciones.

El pensamiento de diseño se ha convertido en un término que se utiliza en grandes compañías como Apple o IBM, en las que se ha implementado para solución de problemas y generación de innovación. «Los procesos de innovación no pueden considerarse como procesos lineales, secuenciales, como tendían a organizarse antes. Hoy en día es preciso un proceso simultáneo con la presencia de todos los implicados, de la misma manera que actúa el cerebro humano en sus procesos creativos» (Colomer, 2012, p. 124).

El pensamiento de diseño, según Liedtka, King y Bennett (2013), es un puente para los líderes, a fin de resolver creativamente problemas por medio de un sistema integral (systematic end-to-end process) y como un reto para innovar. Las soluciones pueden estar enfocadas en diferentes factores como incremento de la productividad, rediseño de procesos internos, aumento en las fuerzas de venta, o lidiar con otro tipo de asuntos; lo importante es que la base siempre será la misma: una metodología para la innovación.

La gestión de la cultura de diseño permite generar soluciones más innovadoras y exitosas, por esta razón, es fundamental entender los elementos que la componen y medirla. Estos elementos se refieren a solucionar problemas complejos, cambio en los servicios y productos, encontrar soluciones más innovadoras, eficientes y efectivas para la organización, una mejor diferenciación de la oferta de productos y servicios, mejorar la comprensión y la relación con el cliente. 
Teniendo en cuenta la intención de esta investigación de demostrar que la cultura del diseño enfocada en la innovación es un elemento esencial para lograr resultados favorables en los procesos de innovación y el éxito de las empresas, fue necesario desarrollar una herramienta que permitiera medir el éxito organizacional que es el resultado propuesto en la figura 1 .

La medición de los beneficios de una cultura de diseño se ha centrado, principalmente, en variables cuantitativas: rentabilidad, atracción de nuevos clientes y competitividad, entre otros. Sin embargo, es muy poco lo que se ha hecho con relación a variables cualitativas. El diseño comúnmente es considerado como intangible (Low y Kalafut, 2002), que, como las comunicaciones y el diseño, realmente se puede medir, y la información para evaluarlo usualmente sí existe.

Lo que se debería medir es qué es lo que —o quién — produce esos resultados. Cómo estos afectan el clima organización y cómo contribuyen a crear una cultura de innovación. Liedtka, King y Bennett (2013) plantean que se debe ver más allá de la punta del iceberg (Figura 3). Se deben buscar los comportamientos que conducen, sistemáticamente, a la rentabilidad, qué comportamientos se asocian con mejores resultados y cómo se puede fomentar la cultura de innovación. 
Figura 3. Cambio en resultados medibles tangibles

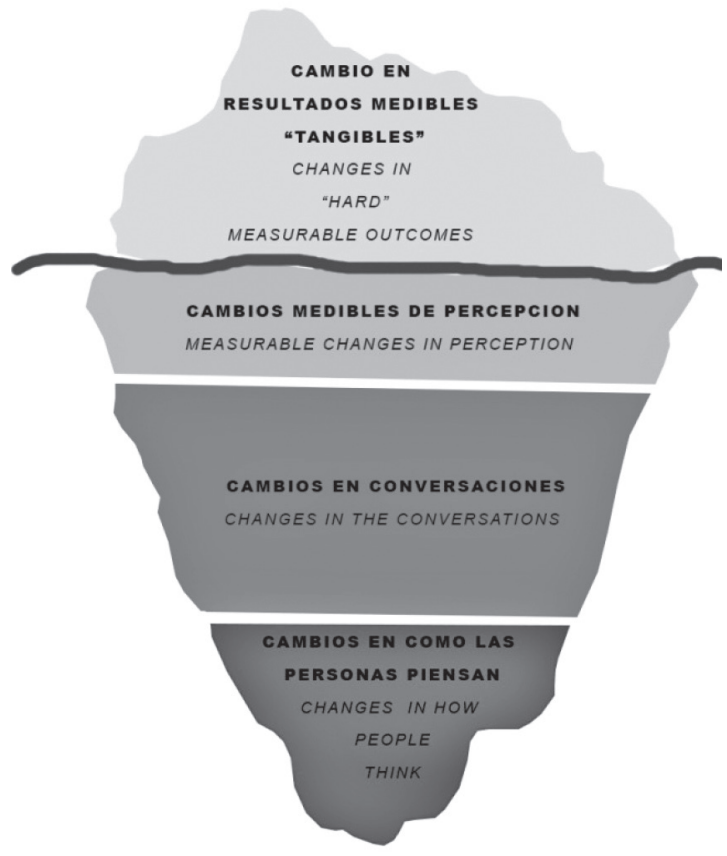

Fuente. Liedtka, King y Bennett, 2013.

Después de todos esos factores tangibles, lo que ocurre son los cambios en las percepciones de la gente, las conversaciones, e incluso la forma de pensar de ellas. Siendo estos cambios más sutiles. Cualquier medición que se haga de estos es perceptiva y subjetiva. Sin embargo, los más significativos son los cambios, no en lo que la gente dice o hace, sino en la manera de pensar y en lo que ellos creen. Incluso, puede haber cambios en cómo las personas piensan sobre sí mismas. Y es ahí donde se puede concebir una cultura de innovación. Liedtka, King y Bennett (2013) exponen casos en los que los comportamientos, las mentalidades y los climas organizacionales han cambiado por medio de las herramientas de DT, tras lo cual se han convertido en una estrategia y ventaja competitiva en sus empresas. 
De tal forma, la investigación se centrará en proponer un modelo que mida la cultura del diseño y sus beneficios, incluso aquellas variables cualitativas y cuantitativas, puesto que «la medición es importante para dirigirse hacia la innovación; puede mejorar e impulsar la eficiencia y hacer del proceso más una ciencia que un arte» ${ }^{10}$ (Lockwood, 2007).

Teniendo en cuenta que el diseño puede actuar como transmisor de los factores organizacionales de las empresas que plantea Hofstede et al. (1990), valores, rituales, héroes y símbolos, la cultura de diseño se podrá medir por medio de estos si se relacionan con los elementos que la componen. Los elementos a los que se hacen referencia son las herramientas que utiliza el pensamiento de diseño para solucionar problemas complejos con un enfoque humano centrista, y que genera innovación.

10 La traducción es mía. 


\section{Modelo para la medición de la cultura de diseño}

L a cultura la componen las prácticas que se llevan a cabo en relación con valores, rituales, héroes y símbolos (Figura 4).

Figura 4. Componentes de la cultura

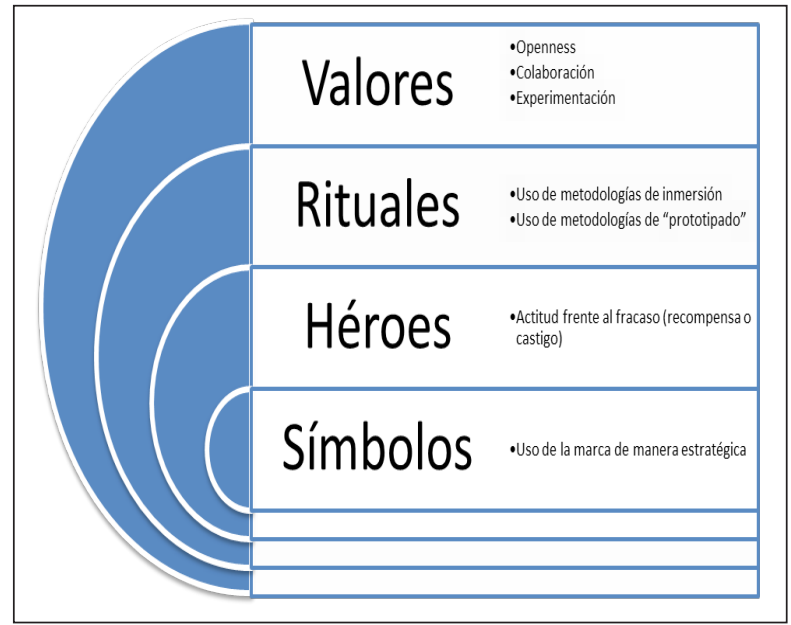

Fuente. Elaboración propia.

\section{5. ¿Cuáles son los valores asociados con la cultura de diseño?}

T a cultura de diseño debe tener la habilidad de ser intuitiva, de $\checkmark$ reconocer patrones, de construir ideas que tienen un significado emocional, pero que al mismo tiempo tienen un sentido funcional, y puedan expresarse en los medios de otras formas a palabras y símbolos (Brown y Wyatt, 2010). 
Los valores asociados con la cultura de diseño deberán tener un pensamiento abierto, colaborativo, de experimentación, de tolerancia al fracaso, siempre priorizando el aprendizaje. Esto se logra por medio de pensamientos divergentes en los que es importante tener un grupo diverso de personas involucrado en los procesos. Personas multidisciplinarias como arquitectos que hayan estudiado Psicología, artistas con MBA, o ingenieros con experiencia en mercadeo- que por lo general demuestran calidad. Son personas con la capacidad y la disposición de colaboración a través de las diferentes disciplinas (Brown y Wyatt, 2010).

Para operar en un ambiente interdisciplinario, un individuo necesita tener fortalezas en dos dimensiones, ser una persona T-shaped. En el eje vertical, cada miembro de un equipo necesita tener un extenso conocimiento de habilidades que le permitan realizar contribuciones tangibles para los resultados. La parte superior de la T es donde el design thinker se crea. Se trata de obtener una empatía hacia las personas y por las disciplinas que van más allá de la propia. Tiende a expresarse como una mentalidad abierta (openness), de curiosidad, optimismo, y una tendencia a aprender a través del hacer y la experimentación (Brown y Wyatt, 2010). Estos valores ya existen en empresas como IDEO, donde se empeñan por contratar a empleados con este perfil. Tim Brown, CEO de IDEO, identifica al design thinker como una persona con «empatía, pensamiento integral, optimista, experimental y colaborativo ${ }^{11}$ (Brown, 2008). Estos valores permiten que se genere innovación dentro de las empresas y en sus productos y servicios.

11 La traducción es nuestra. 


\section{6. ¿Cuáles son los rituales asociados con la cultura de diseño?}

$\mathrm{U}$

na empresa que genera innovación enfocada en las necesidades del cliente concentra varios métodos de innovación a través de la observación de los usuarios. Se localiza en técnicas desarrolladas por profesionales que se engloban bajo el término de diseño centrado en el usuario (Borja, 2006, p. 134). Según Phillips Design, esta época corresponderá a la de un tipo de innovación basada en las personas, y de la creación conjunta con el usuario o del prosumer, abreviatura utilizada para hablar de un usuario coproducto de la oferta. La innovación se genera a partir de la comprensión del valor que estos aspectos asocian a los clientes a las oportunidades de mercado.

De igual forma, la cultura de diseño se caracteriza por ser abierta a proponer o analizar todo tipo de ideas, a generar creatividad. Así mismo, el trabajo en equipo y la colaboración es fundamental para la solución de problemas:

De hecho, la creatividad es un aspecto crucial para obtener la innovación en productos, servicios o procesos, ya que permite integrar el diseño en las actividades principales —o vinculadas a la toma de decisiones - de las empresas, haciendo posible una explotación completa de su potencial innovador. Además, el diseño desarrolla la dimensión conceptual de la generación de ideas: desarrolla conceptos y actúa como integrador de éstos. Estos dos pasos son los detonantes del proceso de innovación. (Carrió, 2006, p. 153) 


\section{7. ¿Los héroes de la cultura de diseño?}

Tos héroes de la cultura de diseño no solo son los líderes, sino aquellos $\smile$ que son más creativos, que se atreven a tomar riesgos y que utilizan su experiencia para enfrontar los problemas. Son aquellos que, como se mencionó, están en la parte superior de la T-shape, con una mentalidad abierta (openness), de curiosidad, optimismo, y una tendencia a aprender a través del hacer y la experimentación. «La creatividad es un aspecto esencial de la innovación: se trata de su punto de partida. Uno de los grandes desafíos de las empresas en la actualidad es el modo de generar más y mejores ideas o, lo que es lo mismo, cómo ser más creativas» (Carrió, 2006, p. 148).

La creatividad no tiene relación con el hecho de tener ideas maravillosas repentinas sin una preparación previa: la creatividad implica relacionar un concepto a un conocimiento adquirido, y dicho conocimiento es tan importante para llegar a la propia idea que las personas realmente creativas pueden invertir muchos años en la tarea de adquirir y refinar esta base de conocimiento (Carrió, 2006).

En paralelo a la existencia de un debate en torno a si la creatividad es un atributo de unos pocos o de todos en general, si bien es cierto que existen individuos más creativos que otros, la creatividad puede estimularse y motivarse a través de formación y favoreciendo el entorno y el ambiente adecuados. Black (1990) enumera las 32 habilidades que, de acuerdo con sus trabajos de investigación, son propias de las personas creativas. Algunos de los que aparecen en la lista no son necesariamente importantes para tener ideas, aunque sí para implementarlas. 
Para ser una empresa competitiva, es necesario contar con individuos que piensen diferente y con otros que se comporten también diferente. Por ello, con frecuencia, la innovación se define como un «marco mental» centrado en la creatividad (Carrió, 2006, p. 151).

\section{8. ¿Cuáles son los símbolos relacionados con la cultura de diseño?}

L

os grupos que alinean sus actividades con las marcas y de los símbolos y su incorporación en los procesos de innovación. «Al diseño se le exige una competencia de imaginación de alianza. La innovación debe ser simultáneamente eficaz y divertida, global y local, industrial y personalizada, tecnológica y poética. El diseño cuenta con una función motriz que debe aplicar en la construcción de la imaginación holística: identidad de marca o esquema narrativo de la innovación» (Borja, 2006, p. 137).

La función motriz del diseño en los cambios de la gestión de la innovación se encuentra en su doble competencia de «usuario»y «holística». Se puede observar el aumento del recurso a los diseñadores estrella en el lanzamiento de productos nuevos y el desarrollo de proyectos interesantes de estrategia de marketing conjunta entre un diseñador reconocido y una marca, de la unión de un estilo estético y de una marca. Algunos diseñadores se convierten a sí mismos en marcas, lo que complica los mecanismos de protección de la innovación (Borja, 2006).

De igual forma, las empresas que se identifican y ponen en práctica la cultura de diseño como, por ejemplo IDEO, no solo generan soluciones, sino que también emociones, y muchas veces las emociones venden más. 
Las soluciones centradas en las personas hacen posible experiencias más allá que un producto como tal. Las experiencias se pueden medir, al igual que diseñar, simplemente al quitar los elementos de diseño previstos, y luego preguntándoles a sus clientes acerca de su experiencia. Estas experiencias crean símbolos dentro y fuera de las organizaciones.

\title{
9. Conclusiones
}

\begin{abstract}
partir de estos resultados, se podrá validar este instrumento. La Amedición llevará a entender en qué medida la cultura de diseño ha ganado importancia en las organizaciones, de qué manera se implementa y quiénes están involucrados. De igual forma, se podrán definir cuáles son los pensamientos sobre el progreso de una empresa si su cultura se basa en el diseño. También interesa entender cuál es el lenguaje utilizado para describir el trabajo relacionado con la cultura y el diseño, como se refleja en los procesos de innovación y en el crecimiento de clientes. ¿Quién habla de estas culturas?
\end{abstract}

Los resultados de la medición también podrán responder inquietudes como: ¿Si se trata de medir el impacto de la cultura de diseño, habrá nuevos cargos de gerencias relacionados con el diseño o la innovación? ¿Se harán departamentos dentro de las empresas enfocados únicamente en la experiencia del usuario? ¿Se incrementarán los roles prominentes desarrollados por las consultorías de diseño? ¿Se contratarán empleados especialistas en gerencia de diseño, particularmente con experiencia y conocimientos de diseño?

Finalmente, debe entenderse quién es el verdadero dueño del diseño. En qué medida los administradores están comprometidos en generar una cultura o pensamiento de diseño. ¿Cómo se puede vender el «diseño» a los ejecutivos de las empresas? 


\section{Referencias}

Borja, B. (2006). El diseño de la innovación, dos retos para la profesión del diseño. Temes de Disseny, 23, 132-146. Recuperado de https://bit. $\mathrm{ly} / 2 \mathrm{HshaC} 3$

Bremner, C. y Rodgers, P. (2013). Design without discipline. Design Issues, 29(3), 4-13. DOI: https://doi.org/10.1162/DESI_a_00217

Brown, T. (2008). Design thinking. Harvard Business Review, 86, 85-92.

Brown, T. y Wyatt, J. (2010). Design thinking for social innovation. Development Outreach, 12(1), 29-43.

Carrió, M. (2006). Espacios comunes del diseño y la innovación. Temes de Disseny, 23, 147-155. Recuperado de https://bit.ly/2YwZ7BK

Colomer, J. (2012). Aplicación del método de escenarios para la conceptualización creativa de productos innovadores en el sector español de baldosas cerámicas (Tesis doctoral). Universitat Politècnica de València, València, España. Recuperado de https://bit.ly/2WMkhLz

Dahi, K. (2015). What design thinking is not about? Recuperado de https://bit. ly/2PPX60e

Design Singapore Council, Ministry of Information, Communications and the Arts (2009). Dsg-II Strategic blueprint of the designsingapore initiative. Singapur.

Hofstede, G., Neuijen, B., Ohayv, D. D. y Sanders, G. (1990). Measuring organizational cultures: A qualitative and quantitative study across twenty cases. Administrative Science Quarterly, 35(2), 286-316. DOI: http:// dx.doi.org/10.2307/2393392

Julier, G. (2010). La cultura del diseño. Barcelona: Gustavo Gili.

Lam, A. (2004). Organizational innovation. Recuperado de https://bit. ly/2H8PC5W 
Liedtka, J., King, A. y Bennett, K. (2013). Solving problems with design thinking: Ten stories of what works. Nueva York: Columbia University Press.

Lockwood, T. (2007). Design value: A framework for measurement. Design Management Review, 18(4), 90-97. DOI: https://doi. org/10.1111/j.1948-7169.2007.tb00099.x

Lockwood, T. (2009). Transition: How to become a more design-minded organization. Design Management Review, 20(3), 28-37. DOI: https://doi. org/10.1111/j.1948-7169.2009.00019.x

Low, J. y Kalafut, P. C. (2002). Invisible advantage: How intangibles are driving business performance. Nueva Yok: Basic Books.

Mora, N. (2012). Innovar para emprender: nuevas metodologías para el emprendimiento. Revista del Colegio Mayor de Nuestra Señora del Rosario, 108(606), 92-98. Recuperado de https://bit.ly/2Wb1mgk

Observatorio Diseño y Empresa (2009). El impacto económico del diseño en las empresas de Cataluña. Recuperado de https://bit.ly/2Lup7fj

Rams, D. (1991). The Munich design charter. Design Issues, 8(1), 74-77. Recuperado de https://bit.ly/2WOWZoj

Reyes, D., Rodríguez, D. Pazcagaza, S. (2014). El diseño en las organizaciones: estudio preliminar de indicadores para su medición. Bogotá: Universidad EAN. 